\title{
Obesidade e saúde bucal: impacto da obesidade sobre condições bucais
}

Obesity and oral health: impact of obesity on oral conditions

\author{
Júlia Machado Saporiti \\ Bruna da Silva Barragana Vera* \\ Breno Soares Arruda** \\ Vanderson de Souza Caldeira** \\ Liz Gill Araujo Pereira* \\ Gustavo Giacomelli Nascimento ${ }^{* * * *}$
}

\section{Resumo}

Objetivo: o objetivo deste estudo foi observar a existência de uma possível relação entre a obesidade e as seguintes doenças bucais: cárie, doenças periodontais e traumatismo dental. Revisão de literatura: a obesidade é considerada uma doença crônica de causa multifatorial, sendo uma complexa associação de fatores genéticos, ambientais, socioeconômicos, biológicos e comportamentais. Sua prevalência vem crescendo entre pessoas de todas as idades, tanto em países de alta renda, quanto em países de baixa e média renda, elencando essa doença como um dos maiores problemas de saúde pública mundial. Além disso, essa condição tem sido apontada como um importante fator de risco a outras doenças como diabetes tipo II, hipertensão, doenças cardiovasculares, câncer, além de problemas psicossociais que afetam diretamente a vida do indivíduo. As doenças bucais também se destacam pela sua etiologia multifatorial e pelo seu desenvolvimento crônico. Essas doenças têm sido apontadas como significantes marcadores biológicos e sociais, e parecem estar ligadas a outras doenças sistêmicas, entre essas, a obesidade. Estudos apontam uma possível associação entre o estado nutricional e a cárie dentária, as doenças periodontais e o traumatismo dentário. Considerações finais: os resultados, entretanto, são controversos entre os diferentes estudos, destacando a ainda existente falta de evidências que suportem essa associação.

Palavras-chave: Obesidade. Cárie dentária. Doenças periodontais.

\section{Introdução}

A obesidade caracteriza-se por danos à condição de saúde causados pelo acúmulo excessivo ou anormal de gordura corpórea ${ }^{1}$. Essa doença não pode ser considerada como uma simples desordem, mas sim, um grupo heterogêneo de condições com múltiplas e complexas causas. O peso corporal é, então, determinado pela interação entre fatores biológicos, ambientais, sociais e comportamentais, agindo na regulação entre o armazenamento e o gasto energético. Diversas são as causas atribuídas à etiologia da obesidade, destacando fatores psicossociais, comportamentais e biológicos. Embora o componente genético tenha um papel inquestionável na etiologia da obesidade, o notável aumento na prevalência dessa condição pode ser mais bem explicado por mudanças sociais e comportamentais, resultados da transição sociodemográfica.

A epidemia da obesidade era uma realidade predominante dos países ricos e desenvolvidos ${ }^{2}$. Ao passo que países de baixa e de média renda começam a se tornar mais globalizados, há uma mudança considerável no padrão nutricional, e nos hábitos de vida, acarretando, consequentemente, uma significante elevação da prevalência dessa doença. Assim, após a década de 1990, a obesidade assume 
taxas nunca antes observadas, e continuadamente crescentes $^{3}$.

O excessivo peso corpóreo é um conhecido fator de risco para inúmeras doenças sistêmicas, como hipertensão, doenças cardiovasculares ${ }^{4}$, diabetes mellitus tipo II $^{5}$ e câncer ${ }^{6}$. As doenças bucais têm etiologia multifatorial, com associação entre fatores biológicos, socioeconômicos e comportamentais ${ }^{7}$. Apresentam natureza crônica e devido à sua alta prevalência estão listadas entre as dez doenças crônicas mais prevalentes do mundo ${ }^{8}$. A literatura tem mostrado uma possível associação entre a obesidade e as doenças bucais, com destaque à cárie dentária, às doenças periodontais e ao traumatismo dentário ${ }^{9-11}$. Entretanto, não há um consenso, na literatura sobre a relação entre o estado nutricional e as doenças bucais, com resultados bem divergentes entre os diferentes estudos.

Baseado nisso, o objetivo deste trabalho foi revisar a literatura, buscando informações a respeito dos possíveis mecanismos envolvidos na relação entre obesidade e cárie dentária, doenças periodontais e traumatismo dentário.

\section{Revisão de literatura}

\section{Obesidade e cárie}

O principal agravo em saúde bucal é a cárie dentária, uma doença crônica, passível de prevenção, de condições cumulativas e considerada como importante causa de dor de origem dentária ${ }^{12}$ e, também, da perda dentária em seu estágio mais avançado ${ }^{13,14}$. A etiologia da cárie dentária é composta por uma complexa interação entre condições biológicas, ambientais e sociais ${ }^{15}$. Embora a prevalência de cárie dentária esteja diminuindo nas últimas décadas, existe uma grande iniquidade na experiência e na distribuição dessa condição no Brasil7.

A literatura científica tem investigado a possível relação entre cárie dentária e obesidade. Entretanto, essa possível associação ainda não está claramente definida, uma vez que os diferentes estudos têm apresentado resultados divergentes em relação a esse assunto. Dentre os estudos conduzidos abordando esse tema, mais de um concorda que existe relação positiva entre cárie e obesidade. Entretanto, a causa dessa relação não está totalmente definida ${ }^{9,16}$. Uma recente revisão sistemática concluiu que a relação entre cárie e obesidade não está clara e que não é possível determinar se realmente há associação entre ambas ou se essas coexistem, já que compartilham etiologia comum e/ou fatores contribuintes similares ${ }^{17}$. Outros estudos relacionam, além da cárie e obesidade, uma relação inversa, em que crianças subnutridas são consideradas mais propensas à carie dental ${ }^{18}$

Os crescentes hábitos sedentários e o aumento da frequência de refeições, particularmente de ali- mentos altamente processados e com grande quantidade de açúcar, têm sido associados com uma dieta não saudável ${ }^{16}$. Esse comportamento sedentário promove um acréscimo do consumo de alimentos que podem aumentar o risco de ganho de peso e da obesidade, e, consequentemente, o maior tempo de contato dos dentes com esses alimentos pode ocasionar o aumento do risco de desenvolvimento de cárie dentária ${ }^{16}$.

Diversos autores mostraram uma correlação positiva entre a cárie dentária e o índice de massa corporal em crianças ${ }^{18,19}$. Indivíduos obesos tendem a consumir alimentos ricos em carboidratos, os quais são altamente cariogênicos ${ }^{17,18}$. Estudos mostraram que o comportamento obeso de ingerir guloseimas na primeira infância prevê o desenvolvimento de cáries na adolescência ${ }^{18}$. Palmer ${ }^{20}$ (2005) mostraram que crianças com alta prevalência de cárie dentária consumiam comidas e bebidas mais frequentemente, se comparadas a crianças livres de cárie. Essa maior frequência de consumo de comidas e bebidas, especialmente a produtos ricos em carboidratos, faz com que haja um incremento do número de microrganismos cariogênicos ${ }^{16,17}$. Somado a isso, o perfil psicológico tende a afetar a propensão do indivíduo a exercer comportamentos benéficos à saúde bucal, como a escovação, o que faz a obesidade ser positivamente associada a uma condição precária de higiene bucal ${ }^{20}$. Assim, o fator emocional pode ter influência nos cuidados com a saúde bucal, pois, em geral, indivíduos obesos tendem a ter uma menor autoestima e, consequentemente, uma pior condição de saúde bucal ${ }^{21}$.

Outro fator que parece estar envolvido na associação entre cárie e obesidade é a composição salivar. Segundo Pannunzio et al. ${ }^{22}$ (2010), crianças obesas apresentam alterações na composição da saliva, o que é favorável ao surgimento de cáries. Essa diminuição do fluxo salivar deve-se ao déficit de proteínas na alimentação, visto que crianças obesas ingerem muitos carboidratos e muitas comidas altamente industrializadas, e poucos alimentos ricos em proteínas ${ }^{18}$.

No estudo conduzido por Hooley et al. ${ }^{18}$ (2012) também é mostrada uma relação inversa, em que a cárie dentária severa pode reduzir a ingestão dos alimentos, o que resulta em perda de peso. Crianças com cárie na primeira infância mostraram alta velocidade de crescimento depois das cáries serem tratadas ${ }^{18}$. Deficiências em alimentos proteicos e energéticos podem levar à má nutrição, diminuindo o fluxo salivar, gerando formação de cálculos, altos níveis de cárie e redução do crescimento. A má nutrição crônica durante os primeiro anos da infância tem sido considerada como fator que aumenta a propensão a cáries na dentição primária ${ }^{17,18}$.

A associação entre cárie com obesidade/sobrepeso tem mostrado uma forte ligação com condição socioeconômica, uma vez que a urbanização e o crescimento econômico alteram de forma significativa 
o padrão alimentar ${ }^{19}$. Assim, o crescimento da taxa de urbanização, somado à melhoria nas condições econômicas populacionais, tem induzido ao aumento no consumo de alimentos altamente processados ricos em carboidratos fermentáveis ${ }^{19}$, tanto nos países de alta quanto nos de baixa e de média renda.

Apesar dessa associação positiva entre cárie dentária e obesidade, alguns autores afirmam que existe uma relação inversa entre esses dois fatores $^{17,18}$. Segundo eles, crianças com menor índice de massa corporal (IMC) tendem a ter mais cáries. Essa afirmação baseia-se na hipótese de que crianças que comem menos consomem mais alimentos ricos em açúcares entre as refeições e, por isso, comem menos da comida principal, a qual deve ser rica em fibras e proteínas ${ }^{17}$. Há também a hipótese de que crianças obesas tenham a ingestão de açúcares diminuída devido à supervisão dos pais, os quais tentam tirar alimentos ricos em carboidratos da alimentação de seus filhos. Alguns autores também defendem que crianças obesas provêm de famílias com melhor status socioeconômico, e, por isso, tem mais acesso aos serviços odontológicos, o que diminui as chances de desenvolvimento da cárie ${ }^{17}$.

De forma semelhante, a baixa condição socioeconômica também parece estar mediando a associação entre cárie dentária e obesidade, uma vez que indivíduos dos estratos sociais mais baixos apresentam maior prevalência de cárie dentária e inadequados hábitos alimentares ${ }^{9,17}$. Alguns autores acreditam que os hábitos de dieta relacionados com obesidade e com baixa renda podem promover uma grande frequência e quantidade do consumo de açúcares ${ }^{9}$.

Assim, devido à complexidade da etiologia da cárie dentária e da obesidade, sua associação permanece incerta. Dessa forma, são necessários estudos com desenho longitudinal para um melhor entendimento da relação entre essas condições.

\section{Obesidade e doenças periodontais}

A doença periodontal (DP) é caracterizada como uma doença crônica, de origem infecciosa, que afeta os tecidos que rodeiam e suportam os dentes ${ }^{23}$. Seu principal agente etiológico é a presença de biofilme bacteriano, todavia inúmeras evidências sugerem que a doença periodontal não está simplesmente associada à colonização da cavidade oral por micro-organismos patogênicos, mas mostra-se ligada à susceptibilidade ou à resistência do hospedeiro, uma vez que, dependendo da resposta imune mediada a esses microrganismos, poderá resultar ou não na destruição do tecido periodontal ${ }^{23}$.

A obesidade, por ser uma enfermidade caracterizada por um acúmulo excessivo de gordura corporal, parece estar intimamente relacionada ao desenvolvimento das doenças periodontais ${ }^{10,20,24}$, embora tal relação ainda não esteja bem explicitada na literatura.
Diversas são as teorias que buscam explicar essa positiva associação entre a obesidade e as doenças periodontais. Recentes estudos sugerem uma associação entre a obesidade e o sistema imune, enquanto que a primeira prejudica a ação efetiva do sistema imune corporal ${ }^{25}$. Esse fator é de suma importância quando se procura entender a ligação entre sobrepeso e doença periodontal, ambas classificadas como doenças crônicas não transmissíveis.

Mesmo diante da descoberta de que a obesidade acomete a imunidade, levando o indivíduo a tornar-se mais propício ao desenvolvimento de doenças periodontais ${ }^{25}$, outros fatores ainda são dúvidas de pesquisadores que procuram entender a relação entre essas duas doenças, como a maior ingestão de açúcares, escovação inadequada e diminuição de secreção salivar em obesos, entre outros.

Estudos recentes apontam a existência de uma associação entre doença periodontal e obesidade ${ }^{26,27}$. Essa relação é representada não somente por fatores biológicos, como também por fatores psicossociais e comportamentais.

Trabalhos realizados demonstram que pessoas com sobrepeso apresentam uma maior incidência de doenças periodontais, como apresentado no seguinte estudo que envolveu crianças entre seis e onze anos de idade, entre os anos de 1999 e 2000, utilizando o IMC como base para tal pesquisa. Nessa pesquisa, observou-se que 30,3\% das crianças estudadas estavam acima do peso e essas apresentavam prevalência de doença periodontal ${ }^{28}$. Esse aumento do índice de DP não afeta apenas crianças, segundo artigos publicados, adultos com sobrepeso também são caracterizados com maior prevalência de doenças periodontais do que adultos em situação normal ou regular de peso ${ }^{28}$.

Foi constatado quando estudado as causas biológicas da relação entre DP e obesidade que o metabolismo celular e tecidual de indivíduos obesos é alterado a nível sistêmico, de forma a levar a uma mudança no desenvolvimento da doença periodontal ${ }^{10}$

Biologicamente, há estudos que apontam o agravamento de citocinas pró-inflamatórias sistêmicas como fator preponderante da relação entre obesidade e $\mathrm{DP}^{4}$. Por sua vez, é de conhecimento geral que a adipocina é uma proteína secretada pelo tecido adiposo branco entre outros, cuja característica funcional é a de alterar a resposta imunológica do organismo, além de ocasionar um estado de inflamação crônico ${ }^{1}$.

Algumas citocinas produzidas por células adiposas e macrófagos, encontrados no processo inflamatório, são de grande relevância no desenvolvimento da periodontite ${ }^{29}$. A leptina e resistina, adipocinas que parecem estar também envolvidas nessa relação, têm a função de manutenção e ativação do processo inflamatório, e tem uma relação com a secreção de citocinas pró-inflamatórias ${ }^{30}$.

Em pacientes obesos e com doença periodontal, os níveis de proteína c-reativa (PCR) encontram-se 
aumentados, levando a constatar que essa está envolvida no processo ${ }^{31}$.

Alguns estudos apontam a presença elevada de marcadores como as adipocinas e o PCR em pessoas obesas com periodontite, que com o tratamento periodontal ou com a perda de massa adiposa acarretam o processo de retroalimentação dessas condições ${ }^{32}$.

Acrescenta-se, também, que o reconhecimento de patógenos pelo organismo e a ação do sistema imune inato são fundamentais para a eliminação do agente patógeno ${ }^{33}$. Mediante esses fatores, o prejuízo da ação do sistema imune inato em indivíduos obesos $^{25}$ pode acarretar um verdadeiro risco de infecções de vários tipos.

Pode-se concluir que devido ao sistema imune prejudicado de indivíduos obesos, infecções como a doença periodontal podem progredir com maior facilidade, visto que a DP pode gerar exposições a endotoxinas lipopolissacarídeas, a bactérias e a produtos bacterianos que influenciam no metabolismo lipídico e na homeostase. Isso ocorre devido à área da superfície total da bolsa de pacientes com doença periodontal ser relativamente grande, podendo comportar $200 \mathrm{mg}$ de biofilme no sulco gengival, acarretando um meio de cultura de microrganismos capaz de invadir a todo o momento os tecidos periodontais subjacentes ${ }^{34}$. A partir disso, ocorre uma pequena faixa de encontro entre a circulação sanguínea e o biofilme bacteriano devido ao comprometimento do epitélio da bolsa periodontal, causando também úlceras em seu interior ${ }^{35}$. Estudos indicam a relação de que quanto maior a severidade da doença periodontal no paciente, mais periodontopatógenos serão encontrados na corrente sanguínea ${ }^{36}$. Notou-se uma íntima relação entre um acúmulo local de bactérias e a escovação dental diária por meio de uma revisão sistemática, não considerando o uso de fio dental independente das condições em que se encontrava a gengiva $^{37}$. Nota-se que ocorreu uma elevação dos valores de mediadores inflamatórios na forma aguda da doença, a qual ocorre devido à natureza cíclica da DP, a casos de aumento e remissão e a constante invasão de bactérias nas bolsas periodontais ${ }^{38}$.

Já é de conhecimento da literatura científica que além de fatores biológicos, fatores socioeconômicos também podem estar envolvidos na relação entre DP e obesidade, visto que a saúde bucal está sendo indicada como um apontador socioeconômico e comportamental, sendo considerada nos estudos referentes à sociedade e à saúde ${ }^{7}$. A prevalência e a severidade das doenças periodontais está ligada ao nível educacional materno e a renda familiar ${ }^{39}$. Em indivíduos com padrão de vida mais baixo observa-se maior prevalência de casos de obesidade ${ }^{40}$. Ambas as doenças apresentam fatores de risco semelhantes como ingestão excessiva de álcool, maus hábitos alimentares, tabagismo e estresse ${ }^{41}$. Uma relação positiva foi encontrada entre estresse psicológico, obesidade e doença periodontal ${ }^{27}$. Esse pri- meiro fator está ligado ao excesso de peso, afetando os hábitos de higiene bucal dos pacientes ${ }^{42}$.

A relação entre emagrecimento e DP é um fator ainda pouco estudado, porém autores tem apontado uma diminuição nos casos de periodontite em indivíduos não obesos ${ }^{43,44}$. $\mathrm{O}$ emagrecimento não gera apenas mudanças biológicas, mas, também, mudanças na alimentação e em hábitos de higiene de modo geral e bucal.

$\mathrm{Na}$ literatura, há apenas um estudo que demonstra a relação de obesidade com sangramento gengival, bolsa periodontal e cálculo dentário em uma coorte de nascimentos ${ }^{26}$. Fez-se um estudo na coorte de nascidos vivos, de 1982 , da cidade de Pelotas, utilizando como instrumentos de medida o IMC. Esse estudo foi realizado com indivíduos da coorte de Pelotas divididos em três períodos de suas vidas: aos 15 anos, aos 18 anos e aos 23 anos. Dois casos de obesidade apontaram a presença de cálculo dental, um fator predisponente à doença periodontal $^{14}$. O presente estudo demonstra alguns limitantes em relação à definição de doença periodontal, sendo que se utilizou o Índice Periodontal Comunitário (CPI) para a determinação da doença. Índice inicialmente criado para medir o nível da população que necessita de tratamento contra a doença periodontal, não apresentando os valores de perda de inserção clínica (CAL) de uma forma concreta. $\mathrm{O}$ único valor registrado consiste na presença de bolsa periodontal, ocorrendo subestimação da doença por não apontar a real severidade e estágio da doença.

Conclui-se, de fato, que é de extrema importância analisar o histórico de obesidade do indivíduo nos vários estágios de sua vida, com dados clínicos de CAL, sangramento à sondagem e cálculo dentário. Vale também ressaltar uma possível predominância da doença em jovens de maioridade, já que a literatura não mostra conhecimentos sobre o assunto.

No contexto dos estudos apresentados, até hoje, sobre o assunto, uma real relação entre a obesidade e às doenças periodontais não pôde ser explicado com clareza. Esse resultado foi consequência, tanto de uma quantidade limitada de pesquisas sobre o tema quanto de uma diversificação de métodos aplicados nos vários estudos. Portanto, mesmo diante de um número significativo de publicações que evidenciam uma relação direta entre a obesidade e a DP, ainda não há evidências longitudinais que possam explicar a possível relação causal entre essas condições.

\section{Obesidade e traumatismo dental}

As lesões dentárias traumáticas são eventos comuns que apresentam uma prevalência entre 2,4 $\%^{45,46}$ e 58,6 \% $\%^{47}$ Nos últimos anos, tem havido crescente preocupação com esse problema de saúde oral, seja pela alta prevalência e pelos custos envolvidos no seu tratamento ${ }^{48}$ seja pelos longos acompanhamentos exigidos. O trauma dental é uma lesão irre- 
versível, que ocorre em várias formas de pequenas fissuras de esmalte à perda do dente ${ }^{49}$.

As lesões dentárias traumáticas são uma das principais emergências infantis, não somente pela prevalência ser expressiva, principalmente, no que se diz respeito a áreas de grande privação social e material $^{50}$, mas, também, por gerar comprometimento à qualidade de vida dos indivíduos com danos irreversíveis à dentição permanente ${ }^{46}$.

Ainda que ocorram outros fatores que aumentem o risco de traumatismo dentário, como: sobressaliência maxilar excessiva, cobertura labial inadequada e alto nível social ${ }^{45}$, são poucos os estudos que envolvem o trauma dentário com o estado nutricional. A maioria dos trabalhos existentes não trazem uma conclusão definida sobre o tema, como pode ser verificado em uma revisão sistemática conduzida por Goettems et $a^{11}$. Os pesquisadores verificaram que há uma divergência na literatura com achados na associação entre Injúria Dental Traumática (Traumatic Dental Injuries) (TDI) e obesidade ${ }^{11}$.

Embora alguns autores tenham afirmado que crianças eutróficas apresentam o mesmo risco para a ocorrência de traumatismo dentário, ${ }^{50}$ outros, ao contrário, encontraram uma associação positiva entre obesidade e maior risco ao trauma dentário ${ }^{51,52}$ Esses autores afirmam que crianças obesas tendem a ser menos ágeis, sendo mais susceptíveis a acidentes, enquanto crianças com peso normal apresentam maior destreza motora, o que, em termos, reduz o risco de quedas acidentais ou incidentes violentos durante a interação com a família e com os amigos $^{47}$ Outro ponto importante a ser considerado é a prática de atividade física entre os indivíduos obesos e os eutróficos. Petti et al. ${ }^{51}$ (1996) afirmam que crianças eutróficas exercitam-se tanto quanto crianças obesas, porém, as últimas, por serem mais desajeitadas, estariam mais propensas a sofrerem traumas. Logo, indivíduos com peso corporal normal são mais hábeis e apresentam menor índice de traumas, por serem sustentados pelo corpo no momento da queda ${ }^{53}$

Além disso, segundo Vijaykumar et al. ${ }^{54}$ (2013), as crianças do sexo masculino com sobrepeso têm uma prevalência maior de trauma quando comparadas às do sexo feminino. Algumas causas que influenciam nesse resultado é que os meninos têm um maior índice de participação em atividades físicas de natureza agressiva. Associado a isso, a alta taxa de gordura corporal diminui a estabilidade e, por isso, as chances de queda aumentam ${ }^{54}$ Os autores ainda observaram que a maior prevalência de traumas em crianças obesas, dar-se-á em ambientes internos por essas se isolarem do resto, devido a deboches e à exclusão do resto do seu grupo escolar.

Ainda que ocorra uma análise mais profunda em artigos de revisão e esses encontrem qual é o fator positivo para a relação entre obesidade e trauma dental, os trabalhos presentes na literatura apontam um delineamento transversal, o que inviabili- za o estabelecimento de uma relação causal entre ambas as condições estudadas, uma vez que o TDI pode ter ocorrido em um momento distinto, não correspondendo com o status nutricional atual. Dessa forma, é essencial que, estudos longitudinais sejam conduzidos para a real compreensão do assunto.

\section{Considerações finais}

Ao final desta revisão, parece haver uma associação entre obesidade e doenças bucais. Entretanto, a maioria dos estudos apresentou um desenho transversal, o que pode ser uma importante limitação para esses achados, uma vez que não é possível o estabelecimento de uma relação causal entre essas condições. Assim, sugere-se que estudos de delineamento longitudinal, envolvendo essa temática sejam desenvolvidos com o objetivo de melhorar a qualidade de vida dos indivíduos e das populações.

\section{Referências}

1. Kopelman PG. Obesity as a medical problem. Nature 2000;404(6778):635-43.

2. Alves LS, Susin C, Dame-Teixeira N, Maltz M. Overweight and obesity are not associated with dental caries among 12-year-old South Brazilian schoolchildren. Community Dent Oral Epidemiol. 2013;41(3):224-31.

3. Popkin BM. Global nutrition dynamics: the world is shifting rapidly toward a diet linked with noncommunicable diseases. Am J Clin Nutr. 2006;84(2):289-98.

4. Chaffee BW, Weston SJ. Association between chronic periodontal disease and obesity: a systematic review and meta-analysis. J Periodontol. 2010;81(12):1708-24.

5. Khader YS, Bawadi HA, Haroun TF, Alomari M, Tayyem $\mathrm{RF}$. The association between periodontal disease and obesity among adults in Jordan. J Clin Periodontol. 2009;36(1):1824.

6. WHO WHO-. Obesity: Preventing and Managing the Global Epidemic. In: Series WTR, editor. Geneva 2000.

7. Oliveira LJ, Correa MB, Nascimento GG, Goettems ML, Tarquinio SB, Torriani DD, et al. [Inequalities in oral health: are schoolchildren receiving the Bolsa Familia more vulnerable?]. Rev. Saúde Pública 2013;47(6):1039-47.

8. Petersen PE, Ogawa H. The global burden of periodontal disease: towards integration with chronic disease prevention and control. Periodontol 2000. 2012;60(1):15-39.

9. Silva AE, Menezes AM, Demarco FF, Vargas-Ferreira F, Peres MA. Obesity and dental caries: systematic review. Rev. Saúde Pública 2013;47(4):799-812.

10. Nascimento GG, Seerig LM, Vargas-Ferreira F, Correa FO, Leite FR, Demarco FF. Are obesity and overweight associated with gingivitis occurrence in Brazilian schoolchildren? J Clin Periodontol. 2013;40(12):1072-8.

11. Goettems ML, Schuch HS, Hallal PC, Torriani DD, Demarco FF. Nutritional status and physical activity level as risk factor for traumatic dental injuries occurrence: a systematic review. Dent Traumatol. 2014.

12. Boeira GF, Correa MB, Peres KG, Peres MA, Santos IS, Matijasevich A, et al. Caries is the main cause for dental pain in childhood: findings from a birth cohort. Caries Res. 2012;46(5):488-95. 
13. Selwitz RH, Ismail AI, Pitts NB. Dental caries. Lancet. 2007;369(9555):51-9.

14. Susin C, Oppermann RV, Haugejorden O, Albandar JM. Tooth loss and associated risk indicators in an adult urban population from south Brazil. Acta Odontol Scand. 2005;63(2):85-93.

15. Thomson WM. Social inequality in oral health. Community Dent Oral Epidemiol. 2012;40 Suppl 2:28-32.

16. Hayden C, Bowler JO, Chambers S, Freeman R, Humphris G, Richards D, et al. Obesity and dental caries in children: a systematic review and meta-analysis. Community Dent Oral Epidemiol. 2013;41(4):289-308.

17. Gonzalez Munoz M, Adobes Martin M, Gonzalez de Dios J. [Systematic review about dental caries in children and adolescents with obesity and/or overweight]. Nutr Hosp. 2013;28(5):1372-83.

18. Hooley M, Skouteris H, Boganin C, Satur J, Kilpatrick N. Body mass index and dental caries in children and adolescents: a systematic review of literature published 2004 to 2011. Syst Rev. 2012;1:57.

19. Honne T, Pentapati K, Kumar N, Acharya S. Relationship between obesity/overweight status, sugar consumption and dental caries among adolescents in South India. Int. J. Dent Hyg. 2012;10(4):240-4.

20. Palmer CA. Dental caries and obesity in children: different problems, related causes. Quintessence Int. 2005;36(6):457-61.

21. Modeer T, Blomberg C, Wondimu B, Lindberg TY, Marcus C. Association between obesity and periodontal risk indicators in adolescents. Int. J. Pediatr. Obes. 2011;6(2-2):264-70.

22. Pannunzio E, Amancio OM, Vitalle MS, Souza DN, Mendes FM, Nicolau J. Analysis of the stimulated whole saliva in overweight and obese school children. Rev. Assoc. Med. Bras. 2010;56(1):32-6.

23. Genco RJ, Grossi SG, Ho A, Nishimura F, Murayama Y. A proposed model linking inflammation to obesity, diabetes, and periodontal infections. J. Periodontol. 2005;76(11 Suppl):2075-84.

24. Fadel HT, Pliaki A, Gronowitz E, Marild S, Ramberg P, Dahlen $\mathrm{G}$, et al. Clinical and biological indicators of dental caries and periodontal disease in adolescents with or without obesity. Clin Oral Investig. 2013

25. Schmidt AM, Weidman E, Lalla E, Yan SD, Hori O, Cao R, et al. Advanced glycation endproducts (AGEs) induce oxidant stress in the gingiva: a potential mechanism underlying accelerated periodontal disease associated with diabetes. J Periodontal Res. 1996;31(7):508-15.

26. Castilhos ED, Horta BL, Gigante DP, Demarco FF, Peres KG, Peres MA. Association between obesity and periodontal disease in young adults: a population-based birth cohort. J. Clin Periodontol. 2012;39(8):717-24.

27. Reeves AF, Rees JM, Schiff M, Hujoel P. Total body weight and waist circumference associated with chronic periodontitis among adolescents in the United States. Arch Pediatr Adolesc Med. 2006;160(9):894-9.

28. Arslanian S, Suprasongsin C. Insulin sensitivity, lipids, and body composition in childhood: is "syndrome X" present? $\mathrm{J}$ Clin Endocrinol Metab. 1996;81(3):1058-62.

29. Preshaw PM, Taylor JJ. How has research into cytokine interactions and their role in driving immune responses impacted our understanding of periodontitis? J Clin Periodontol. 2011;38 Suppl 11:60-84

30. Zimmermann GS, Bastos MF, Dias Goncalves TE, Chambrone L, Duarte PM. Local and circulating levels of adipocytokines in obese and normal weight individuals with chronic periodontitis. J Periodontol. 2013;84(5):624-33.
31. Bullo M, Garcia-Lorda P, Megias I, Salas-Salvado J. Systemic inflammation, adipose tissue tumor necrosis factor, and leptin expression. Obes Res. 2003;11(4):525-31.

32. Zuza EP, Barroso EM, Carrareto AL, Pires JR, Carlos IZ, Theodoro LH, et al. The role of obesity as a modifying factor in patients undergoing non-surgical periodontal therapy. J. Periodontol. 2011;82(5):676-82.

33. Karlsson EA, Beck MA. The burden of obesity on infectious disease. Exp Biol Med (Maywood). 2010;235(12):1412-24.

34. Socransky SS, Smith C, Martin L, Paster BJ, Dewhirst FE, Levin AE. "Checkerboard" DNA-DNA hybridization. Biotechniques 1994;17(4):788-92.

35. Hujoel PP, Cunha-Cruz J, Kressin NR. Spurious associations in oral epidemiological research: the case of dental flossing and obesity. J Clin Periodontol. 2006;33(8):520-3.

36. Silver JG, Martin AW, McBride BC. Experimental transient bacteraemias in human subjects with varying degrees of plaque accumulation and gingival inflammation. J. Clin Periodontol. 1977;4(2):92-9.

37. Tomas I, Alvarez M, Limeres J, Potel C, Medina J, Diz P. Prevalence, duration and aetiology of bacteraemia following dental extractions. Oral Dis. 2007;13(1):56-62.

38. Wu T, Trevisan M, Genco RJ, Falkner KL, Dorn JP, Sempos CT. Examination of the relation between periodontal health status and cardiovascular risk factors: serum total and high density lipoprotein cholesterol, C-reactive protein, and plasma fibrinogen. Am J Epidemiol. 2000;151(3):273-82.

39. Thomson WM, Broadbent JM, Poulton R, Beck JD. Changes in periodontal disease experience from 26 to 32 years of age in a birth cohort. J Periodontol. 2006;77(6):947-54.

40. Gibbs BG, Forste R. Socioeconomic status, infant feeding practices and early childhood obesity. Pediatr Obes. 2013.

41. Genco RJ, Borgnakke WS. Risk factors for periodontal disease. Periodontol 2000. 2013;62(1):59-94.

42. Mellin AE, Neumark-Sztainer D, Story M, Ireland M, Resnick MD. Unhealthy behaviors and psychosocial difficulties among overweight adolescents: the potential impact of familial factors. J Adolesc Health 2002;31(2):145-53.

43. Pataro AL, Costa FO, Cortelli SC, Cortelli JR, Dupim Souza AC, Nogueira Guimaraes Abreu MH, et al. Influence of obesity and bariatric surgery on the periodontal condition. J. Periodontol. 2012;83(3):257-66.

44. Lakkis D, Bissada NF, Saber A, Khaitan L, Palomo L, Narendran S, et al. Response to periodontal therapy in patients who had weight loss after bariatric surgery and obese counterparts: a pilot study. J Periodontol. 2012;83(6):684-9.

45. Grimm S, Frazao P, Antunes JL, Castellanos RA, Narvai PC. Dental injury among Brazilian schoolchildren in the state of Sao Paulo. Dent Traumatol. 2004;20(3):134-8.

46. Alonge OK, Narendran S, Williamson DD. Prevalence of fractured incisal teeth among children in Harris County, Texas. Dent Traumatol. 2001;17(5):218-21.

47. Marcenes W, Murray S. Social deprivation and traumatic dental injuries among 14-year-old schoolchildren in Newham, London. Dent Traumatol. 2001;17(1):17-21.

48. Borum MK, Andreasen JO. Therapeutic and economic implications of traumatic dental injuries in Denmark: an estimate based on 7549 patients treated at a major trauma centre. Int. J. Paediatr Dent. 2001;11(4):249-58.

49. Schuch HS, Goettems ML, Correa MB, Torriani DD, Demarco FF. Prevalence and treatment demand after traumatic dental injury in South Brazilian schoolchildren. Dent Traumatol. 2013;29(4):297-302. 
50. Nicolau B, Marcenes W, Sheiham A. The relationship between traumatic dental injuries and adolescents' development along the life course. Community Dent Oral Epidemiol. 2003;31(4):306-13.

51. Petti S, Tarsitani G. Traumatic injuries to anterior teeth in Italian schoolchildren: prevalence and risk factors. Endod Dent Traumatol. 1996;12(6):294-7.

52. Petti S, Cairella G, Tarsitani G. Childhood obesity: a risk factor for traumatic injuries to anterior teeth. Endod Dent Traumatol. 1997;13(6):285-8.

53. Soriano EP, Caldas Ade F, Jr., Diniz De Carvalho MV, Amorim Filho Hde A. Prevalence and risk factors related to traumatic dental injuries in Brazilian schoolchildren. Dent Traumatol. 2007;23(4):232-40.

54. Vijaykumar S, Guna Shekhar M, Vijayakumar R. Traumatic dental injuries and its relation to overweight among indian school children living in an urban area. J Clin Diagn Res. 2013;7(11):2631-3.

Endereço para correspondência:

Júlia Machado Saporiti

Pe. Anchieta, no 1417/512

96015-420 Pelotas-RS

E-mail: julia.saporiti@hotmail.com

Recebido: 10/08/2014. Aceito: 05/12/2014. 